\title{
On the Normalization of a Mass Spectrum for Comparison of Two Spectra
}

\author{
Zeev B. Alfassi \\ Department of Nuclear Engineering, Ben Gurion University, Beer Sheva, Israel
}

It is indicated that for comparison of two mass-spectra the normalization of each ion current should be done such that each spectrum will form a unit vector, i.e., $\Sigma X_{i}^{2}=1$, where $X_{i}$ are the various components of the mass spectrum. (J Am Soc Mass Spectrom 2004, 15, 385-387) (C) 2004 American Society for Mass Spectrometry

$\mathrm{W}$ hen comparing the mass-spectra of two compounds or the mass spectrum of an unknown compound with a library collection of massspectra, the raw data, i.e., that of the ions intensities of the various $\mathrm{m} / \mathrm{z}$ peaks, cannot be used directly. The amounts of the unknown and the standards might be different due to various physical and chemical reasons leading to different total ion currents. Therefore, the comparison usually can be done only after normalization of the ion currents.

\section{Discussion}

Rasmussen and Isenhour [1] tested the usefulness of several testing algorithms and suggested several normalization methods. In the first method they divide the currents of all ions by the current of the $\mathrm{m} / \mathrm{z}$ with the highest current (base peak normalization). In the second normalization method, the sum of the currents of all the ions is taken as unity, i.e., every $\mathrm{m} / z$ current $I_{i}$ is normalized by dividing by $\Sigma I_{i}$, which means taking the relative intensities. The third normalization factor is $\Sigma I_{i}^{2}$, which leads to each spectrum being a vector of unit length. They studied the use of these three normalization methods for several metrics (i.e., criteria) to compare the spectrum of an unknown compound with a collection of mass spectra in a library. Rasmussen and Isenhour [1] found that all the normalization methods and search methods gave similar results, although the total ion current normalization seems the to be best normalization method.

Stein and Scott [2] gave a clearer presentation of the search methods. They suggested looking at the intensities of different $\mathrm{m} / \mathrm{z}$ as the components of a vector and normalizing the vectors to unit length. Each individual normalized vector can be looked at as a single point on

Published online January 14, 2004

Address reprint requests to Dr Z. B. Alfassi, Department of Nuclear Engineering, Ben Gurion University, Beer Sheva 84105, Israel. E-mail: alfassi@bgumail.bgu.ac.il a sphere with unit radius in a hyperspace of $n$ dimensions, where $n$ is the number of components of the vector. If two mass-spectra (points on the $n$-dimensional hypersphere) are identical in all the values of the components they will be a perfect "match" and will be the same point in the hyperspace. However, due to instrumental variability and instability and because of the statistical nature of the measurements, very rarely will the point of the unknown coincide with one point of the library of standards. They suggested looking at the problem of finding possible matches as the determination of which standard points will be within a minimal volume element centered at the point of the unknown (i.e., in a minimal distance from the point characteristic to the unknown). The similarity of two normalized vectors can be seen as the inverse of their distance apart. However, Stein and Scott's definition of the Euclidean geometric matching factor:

$$
M F_{g}=\left[1+\frac{\sum\left(u_{i}-s_{i}\right)^{2}}{\sum u_{i}^{2}}\right]^{-1}
$$

where $\mathrm{u}_{i}$ and $\mathrm{s}_{i}$ are the $i$-th component of the unknown sample and the standard one from the library, respectively, show that they did not follow their own reasoning when they wrote this equation. There is no reason to normalize both the spectra of the unknown and the library standards according to the length of the vector of the unknown. In order to find the right geometrical distance, each vector (both the unknown and the library collection) must be normalized separately, as each one of them should have a unit length in order to be on this hyperspace. The correct equation for the Euclidean geometric distance matching factor should be:

$$
M F_{g}=\left[1+\sum\left(\frac{u_{i}}{\sqrt{\sum u_{i}^{2}}}-\frac{s_{i}}{\sqrt{\sum s_{i}^{2}}}\right)^{2}\right]^{-1}
$$

The addition of $1+$ in square brackets to the real 
geometric distance in the hyperspace was to prevent having a zero denominator in the case of identical vectors and to bring the range of the matching factor to be from one to zero, when 1 means perfect identification-identical vectors. Stein and Scott found that their Euclidean-distance matching factor (eq 1) yields only $69.8 \%$ of "first hits", while the matching factor of scalar product, i.e., the calculation of $\cos ^{2} \theta$, yields $73.2 \%$ of first hits. This error in normalization explains why Stein and Scott [2] found differences in the number of hits when comparing the geometrical matching factor with the $\cos ^{2} \phi$ matching factor (from the scalar product of the vectors), although they themselves wrote that there is a mathematical correlation between the geometrical distance (d) and $\cos ^{2} \phi$ :

$$
\begin{aligned}
& d^{2}=1^{2}+1^{2}-2 \cdot 1 \cdot 1 \cdot \cos \theta \\
& d^{2}=2(1-\cos \theta)
\end{aligned}
$$

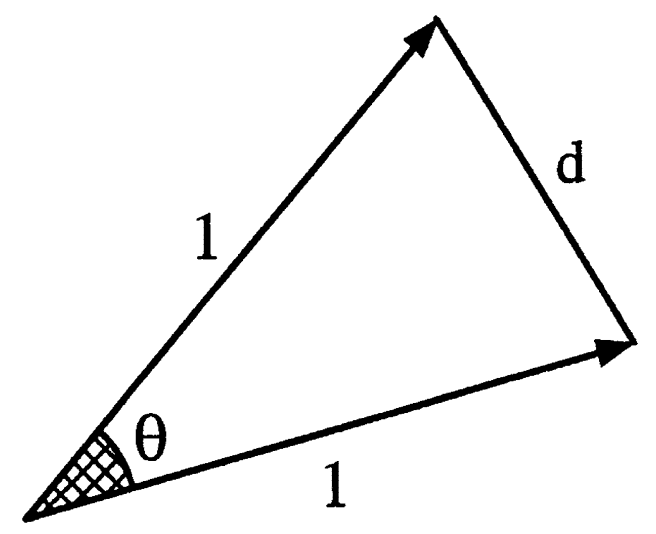

$$
\cos \theta=1-0.5 d^{2}
$$

Since the geometric distance (d) is one to one correlated with $\cos ^{2} \phi$ it is not possible that they will lead to different ordering of the library spectra and hence they must lead to the same number of hits. The only reason for the different number of hits is the wrong equation that Stein and Scott used for the geometrical distance (d). In order to prove that the use of the correct equation (eq 2) will lead to the same number of hits as with the use of the $\cos ^{2} \theta$, and since we do not have the data of Stein and Scott, we checked it in another system. We utilize the same method of multi-parameter analysis in order to find the location of a small radioactive material in the lung by using the counts from four $\gamma$-detectors [5, 6]. The readings of the four detectors are the components of our vector and it is compared to the vectors of known standards. It was found that in all cases the number of hits obtained by eq 2 is equal to that obtained by the $\cos ^{2} \theta$ method. In all our studies eq 1 yields less number of hits than eq 2 , indicating on the preference of eq 2.

Gross and coworkers started with normalizing in relation to only one of the vectors [3] (or more accurately each component was normalized to the lower value of this component in the two spectra) and then changed to normalization with respect to the arithmetic mean of the two vectors [4]. However, even this cooperative normalization does not have any physical or mathematical meaning. Why use the arithmetic mean? Why not use the geometric or the harmonic one? This kind of normalization does not solve correctly the possibility that one of the pair spectra has higher total current due to either different pressures or different ionization efficiencies in the different systems. Normalization of each vector to unit length cancels all these effects.

The normalization of each vector alone actually also simplifies the calculation for the comparison of the mass spectrum of an unknown compound with the library of mass-spectra of known compounds. The mass spectra of the known compounds should be normalized only once, keeping in the stored library only normalized vectors. Any comparison of the mass spectrum of an unknown compound will require only the normalization of this compound independent how large is our library.

Testing by eq 2 , it is equal to testing by the scalar product, $\cos ^{2} \theta$ test, and although the $\cos ^{2} \phi$ test does not require, in principle, any normalization as it does not measure the distance but the deviation from parallelism, the use of a normalized vector reduces the amount of computations. While $\cos ^{2} \theta$ in the case of un-normalized vector is given by the equation

$$
\cos ^{2} \Phi=\frac{\sum X_{i} Y_{i}}{\sum\left(X_{i}^{2}\right) \cdot \Sigma\left(Y_{i}\right)^{2}}
$$

in the case of normalized vectors the equation is much simpler and require less computations.

$$
\cos ^{2} \Phi=\Sigma X_{i} Y_{i}
$$

In the case of storing the library data as normalized vectors, we have to calculate the normalization of each vector in the library only once before storing it in the library. No further normalization is required if correct normalization is done before storing as we can use eq 5 . If the library vectors are not normalized or normalized incorrectly, we must use eq 4 , which means that we are computing the normalization of all vectors every time we are calculating $\cos ^{2} \theta$.

In summary, normalizing all vectors to unit length gives both more physical meaning and less computation time. 


\section{References}

1. Rasmussen, G. T.; Isenhour, T. L. The Evaluation of Mass Spectral Search Algorithm. J. Chem. Inf. Comput. Sci. 1979, 19, 179-186.

2. Stein, S. E.; Scott, D. R. Optimization and Testing of Mass Spectra Library Search Algorithms for Compound Identification. J. Am. Soc. Mass Spectrom. 1994, 5, 859-866.

3. Lay, J. O.; Gross, M. L.; Zwinselman, J. J.; Nibbering, N. M. M. A Field Ionization and Collisionally Activated Dissociation/
Charge Stripping Study of Some $\left[\mathrm{C}_{9} \mathrm{H}_{10}\right]^{+\cdot}$ Ions. Org. Mass Spectrom. 1983, 18, 16-21.

4. Wan, K. X.; Vidavsky, I.; Gross, M. L. Comparing Similar Spectra: From Similarity Index to Spectral Contrast Angle. J. Am. Soc. Mass Spectrom. 2002, 13, 85-88.

5. Pelled, O.; German, U.; Pollak, G.; Alfassi, Z. B. Estimation of Errors Due to Inhomogeneous Distribution of Radionuclides in Lungs. Proceedings of the European IRPA Congress; Florence, Italy, 2002.

6. Pelled, O.; Tzroya , S.; German, U.; Haquin, G.; Alfassi, Z. B.; Locating a "Hot Spot" in the Lungs When Using an Array of Four HPGe Detectors, unpublished. 